\title{
Desempenho de cordeiros alimentados com inclusão de torta de macaúba na dieta
}

\author{
Rafael Alves de Azevedo(1), Luana Marta de Almeida Rufino(1), Antonio Carlos Ramos dos Santos(1), \\ Lígia Pires da Silva ${ }^{(1)}$, Hugo Colombarolli Bonfá(1), Eduardo Robson Duarte ${ }^{(1)}$ e Luciana Castro Geraseev(1) \\ (1)Universidade Federal de Minas Gerais, Instituto de Ciências Agrárias, Avenida Universitária, no 1000, Bairro Universitário, \\ CEP 39404-547 Montes Claros, MG. Email: rafaelzooufmg@gmail.com, Imarufino@yahoo.com.br, antoniocarlosrs@zootecnista.com.br, \\ liggias_silva@hotmail.com, hugocbonfa@hotmail.com, duartevet@hotmail.com, Igeraseev@gmail.com
}

\begin{abstract}
Resumo - O objetivo deste trabalho foi avaliar o consumo de nutrientes e o desempenho produtivo e econômico de ovinos confinados da raça Santa Inês, alimentados com dietas com diferentes taxas de inclusão de torta de macaúba. Os animais (24) foram distribuídos em delineamento de blocos ao acaso, nos tratamentos com 0 , 100,200 e $300 \mathrm{~g} \mathrm{~kg}^{-1}$ de torta de macaúba na matéria seca da dieta, em seis repetições, durante 60 dias de confinamento. A adição de torta de macaúba a taxas crescentes à dieta aumentou linearmente o consumo de proteína bruta, extrato etéreo, fibra em detergente neutro e a conversão alimentar. Não houve diferença no consumo de matéria seca, ganho médio diário e ganho de peso vivo total com a adição da torta. $\mathrm{O}$ tratamento com inclusão de $100 \mathrm{~g} \mathrm{~kg}^{-1}$ apresentou a melhor relação custo:benefício na avaliação das receitas bruta e líquida obtidas com a venda dos animais vivos e abatidos. A adição da torta de macaúba em até $300 \mathrm{~g} \mathrm{~kg}^{-1}$ da dieta não altera o desempenho produtivo dos animais, entretanto, a inclusão de $100 \mathrm{~g} \mathrm{~kg}^{-1}$ proporciona melhor retorno econômico.
\end{abstract}

Termos para indexação: Acrocomia aculeata, confinamento, coproduto, ovinos, terminação.

\section{Performance of lambs fed with macauba pie inclusion in the diet}

\begin{abstract}
The objective of this work was to evaluate the nutrient uptake and the productive and economic performance of confined Santa Inês lambs fed diets with different levels of macauba pie inclusion. The animals (24) were distributed in a randomized block design, in treatments with $0,100,200$ and $300 \mathrm{~g} \mathrm{~kg}^{-1}$ macauba pie in the diet dry matter, with six replicates during 60 days of confinement. The addition of macauba pie at increasing rates to the diet linearly increased the intake of crude protein, ether extract, neutral detergent fiber and feed conversion. There was no difference in dry matter intake, average daily gain and total live weight gain with the macauba pie addition. Diet with $100 \mathrm{~g} \mathrm{~kg}^{-1}$ macauba pie inclusion showed the best cost:benefit ratio in the evaluation of gross and net revenues from the sale of live and slaughtered animals. The addition of macauba pie up to $300 \mathrm{~g} \mathrm{~kg}^{-1}$ to the diet does not affect the animal growth performance; however, the addition of $100 \mathrm{~g} \mathrm{~kg}^{-1}$ macauba pie provides a better economic return.
\end{abstract}

Index terms: Acrocomia aculeata, feedlot, by-product, sheep, termination.

\section{Introdução}

O Brasil possui um rebanho ovino expressivo, que ultrapassa dezessete milhões de cabeças (Food and Agriculture Organization of the United Nations, 2010). Desse rebanho, $56 \%$ encontra-se na região Nordeste (Instituto Brasileiro de Geografia e Estatística, 2010), que é caracterizada pela adoção de sistema de produção extensivo, com baixos índices zootécnicos, abate de animais tardios, qualidade de carne incompatível com a demanda, além da irregularidade de oferta do produto (Nunes et al., 2007).

Segundo Macedo et al. (2007), é necessário avaliar sistemas mais eficientes, com o objetivo de reduzir a idade ao abate e produzir carcaças de melhor qualidade. Sistemas modernos de criação utilizam manejos e opções alimentares adequadas para promover melhor desempenho zootécnico dos animais e maior retorno econômico (Cardoso et al., 2006). Assim, o interesse pela utilização de coprodutos agroindustriais, na alimentação de ruminantes, tem sido fomentado em razão de questões ambientais e econômicas.

A torta da macaúba, resíduo da extração do óleo da polpa do fruto, pode ser um coproduto viável para a alimentação de ovinos, principalmente em locais como a região do Semiárido brasileiro, em razão da disponibilidade da palmeira [Acrocomia aculeata (Jacq.) Lodd. Ex Mart.]. Análises químicas preliminares,

Pesq. agropec. bras., Brasília, v.47, n.11, p.1663-1668, nov. 2012 
realizadas nas tortas residuais do coco da macaúba, mostram que este coproduto poderia ser aproveitado na alimentação de ruminantes, principalmente pelo maior teor de gordura em comparação ao milho (Barreto, 2008). Entretanto, não há trabalhos na literatura sobre a utilização desse resíduo na alimentação de ovinos e, portanto, são necessárias pesquisas para avaliar os efeitos da utilização sobre o desempenho dos animais.

O objetivo deste trabalho foi avaliar o consumo de nutrientes e o desempenho produtivo e econômico de ovinos confinados da raça Santa Inês, alimentados com dietas com diferentes taxas de inclusão de torta de macaúba.

\section{Material e Métodos}

O experimento foi conduzido no setor de ovinocultura do Instituto de Ciências Agrárias, da Universidade Federal de Minas Gerais (ICA/UFMG), em Montes

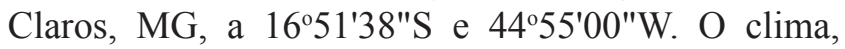
segundo classificação de Köppen-Geiger, é do tipo Aw, considerado tropical de savana, com longo período seco e verão chuvoso.

Foram utilizados 24 cordeiros da raça Santa Inês, machos, não castrados, com idade média de cinco meses e com peso vivo médio inicial (PVI) de $23,9 \pm 0,6 \mathrm{~kg}$. Os animais foram distribuídos em delineamento de blocos ao acaso, com quatro tratamentos e seis repetições. Os blocos foram definidos de acordo com o peso corporal inicial; no primeiro bloco, a média de PVI foi de 20,9 $\mathrm{kg}$, no segundo, $23,7 \mathrm{~kg}$ e, no terceiro, $27,0 \mathrm{~kg}$. Os animais foram confinados em baias individuais de $1,2 \mathrm{~m}^{2}$, providas com comedouros e bebedouros. Adotaram-se procedimentos de acordo com os princípios éticos de experimentação animal, conforme protocolo 87/2008, aprovado pelo Comitê de Ética em Experimentação Animal da Universidade Federal de Minas Gerais.

O experimento teve duração de 70 dias, tendo sido os 10 primeiros destinados à adaptação dos animais ao ambiente e à dieta, e os 60 dias restantes para coletas dos dados. No período pré-experimental, os animais foram pesados, identificados, tratados com o vermífugo Aldazol $10 \mathrm{CO}$ e a vacina Poli-Star contra clostridiose, ambos da Vallée S.A., São Paulo, SP, Brasil.

As dietas experimentais foram formuladas de acordo com as recomendações do National Research Council (2007), para ganho médio de peso de 200 g por dia, com quantidades crescentes de TM $(0,100,200$ e $300 \mathrm{~g} \mathrm{~kg}^{-1}$ da MS) (Tabela 1).

Os animais receberam dietas completas com $300 \mathrm{~g} \mathrm{~kg}^{-1}$ de volumoso (silagem de sorgo) e $700 \mathrm{~g} \mathrm{~kg}^{-1}$ de concentrado (milho, farelo de soja, caroço de algodão, torta de macaúba, calcário calcítico, fosfato

Tabela 1. Composição das dietas utilizadas na alimentação de cordeiros Santa Inês.

\begin{tabular}{|c|c|c|c|c|c|}
\hline \multirow{2}{*}{$\begin{array}{l}\text { Ingredientes } \\
\left(\mathrm{g} \mathrm{kg}^{-1} \text { de matéria seca) }\right.\end{array}$} & \multicolumn{4}{|c|}{ Inclusão de torta de macaúba ( $\mathrm{g} \mathrm{kg}^{-1}$ de matéria seca) } & \multirow{2}{*}{$\begin{array}{l}\text { Torta de } \\
\text { macaúba }\end{array}$} \\
\hline & 0 & 100 & 200 & 300 & \\
\hline Silagem de sorgo & 300,00 & 300,00 & 300,00 & 300,00 & - \\
\hline Farelo de soja & 149,80 & 153,00 & 156,10 & 159,30 & - \\
\hline Milho & 486,50 & 384,00 & 281,40 & 178,90 & - \\
\hline Torta de macaúba & 00,00 & 100,00 & 200,00 & 300,00 & - \\
\hline Caroço de algodão & 50,00 & 50,00 & 50,00 & 50,00 & - \\
\hline Fosfato bicálcico & 03,30 & 03,30 & 03,40 & 03,40 & - \\
\hline Calcário & 07,30 & 06,70 & 06,10 & 05,40 & - \\
\hline Sal comum & 02,80 & 02,80 & 02,80 & 02,80 & - \\
\hline Premix & 00,20 & 00,20 & 00,20 & 00,20 & - \\
\hline Matéria seca & 729,20 & 739,40 & 743,00 & 748,00 & 949,60 \\
\hline Proteína bruta & 150,10 & 158,80 & 158,10 & 157,40 & 81,80 \\
\hline Extrato etéreo & 39,00 & 49,50 & 62,00 & 67,90 & 149,50 \\
\hline Matéria mineral & 42,80 & 46,80 & 51,90 & 58,60 & 34,50 \\
\hline Fibra em detergente ácido & 167,50 & 203,30 & 249,60 & 272,30 & 493,90 \\
\hline Fibra em detergente neutro & 320,90 & 363,50 & 408,80 & 431,70 & 620,30 \\
\hline Carboidratos não fibrosos & 447,20 & 381,40 & 319,20 & 284,40 & 113,90 \\
\hline Nutrientes digestíveis totais $^{(1)}$ & 663,40 & 653,70 & 645,90 & 639,80 & 681,00 \\
\hline
\end{tabular}

(1)Nutrientes digestíveis totais $=40,2625+(0,1969 \mathrm{x}$ proteína bruta $)+(0,4028 \mathrm{x}$ teores de carboidratos não fibrosos $)+(1,903 \mathrm{x}$ extrato etéreo $)-(0,1379 \mathrm{x}$ fibra em detergente neutro) (Weiss, 1998). 
bicálcico e premix mineral e vitamínico). A torta da macaúba (TM) utilizada foi doada pela Cooperativa de Pequenos Produtores Rurais de Riacho D'Antas e Adjacências, localizada em Montes Claros, MG.

A alimentação foi fornecida duas vezes ao dia, às 7 $\mathrm{h}$ e às $17 \mathrm{~h}$, e ajustada de forma a manter as sobras em $200 \mathrm{~g} \mathrm{~kg}^{-1}$ do oferecido. Essas sobras foram retiradas e pesadas diariamente, para determinação do consumo diário. Os consumos dos nutrientes foram expressos em gramas por dia, gramas por quilo de peso corporal ( $\mathrm{g}$ por $\mathrm{kg}$ PC) e por unidade de peso metabólico (g por $\mathrm{PV}^{-0,75}$ ).

Amostras dos alimentos fornecidos e das respectivas sobras foram coletadas diariamente, identificadas e acondicionadas em freezer. Todas as amostras foram pré-secadas em estufa ventilada a $55^{\circ} \mathrm{C}$ e, posteriormente, moídas em moinho com peneira de $1 \mathrm{~mm}$ e acondicionadas em frascos identificados, para posteriores análises laboratoriais.

As amostras das dietas e das sobras foram analisadas quanto ao conteúdo em matéria seca (MS), matéria mineral $(\mathrm{MM})$, proteína bruta $(\mathrm{PB})$, extrato etéreo (EE), fibra em detergente neutro (FDN) e fibra em detergente ácido (FDA), conforme Silva \& Queiroz (2002). Em todas as amostras, os teores de carboidratos não fibrosos (CNF) foram quantificados de acordo com a equação de Weiss (1998): $\mathrm{CNF}(\%)=100-(\% \mathrm{~PB}+\% \mathrm{EE}+\% \mathrm{MM}+\% \mathrm{FDN})$. A ingestão de alimentos foi calculada pela diferença diária dos pesos do alimento oferecido e das sobras.

As pesagens dos animais foram realizadas a cada sete dias, sempre no mesmo horário, antes da primeira refeição. Ao completar 60 dias do período experimental, os animais foram pesados para obtenção do peso vivo final (PVF), ganho médio diário (GMD), ganho de peso vivo total (GPVT) e conversão alimentar (CA).

Ao final do experimento, os animais foram submetidos a jejum de sólidos por 14 horas e, em seguida, foram abatidos. $\mathrm{O}$ abate foi realizado por atordoamento, seguido de sangria por $5 \mathrm{~min}$, com corte da carótida e jugular. As carcaças foram limpas, evisceradas e levadas para uma câmara de refrigeração, tendo permanecido penduradas pela articulação tarso-metatarsiana em ganchos distanciados de $17 \mathrm{~cm}$ por período de 24 horas a $5^{\circ} \mathrm{C}$. Depois de resfriadas, foram pesadas para a obtenção do peso da carcaça fria (PCF).
A análise econômica teve como objetivo avaliar a viabilidade econômica da inclusão da TM na alimentação dos cordeiros confinados. Portanto, não foram considerados, no presente estudo, os custos fixos e operacionais relativos aos itens mão de obra, sanidade e despesas diversas. Nesta análise, foram considerados preços de mercado para os ingredientes da dieta, valor de aquisição dos animais e valores obtidos com a venda do cordeiro. Com o custo de cada ração e do consumo, foi calculado o resultado econômico proporcionado por cada dieta.

As análises estatísticas foram interpretadas por meio de análises de variância e de regressão pelo sistema de análises estatísticas e genéticas SAEG (2007).

\section{Resultados e Discussão}

Não houve efeito $(p>0,05)$ das quantidades adicionadas de torta de macaúba (TM) sobre o consumo de MS e de MO (Tabela 2).

Os resultados de consumo de MS variaram de 1.093 a $1.179 \mathrm{~g}$ por dia. O consumo observado foi superior ao CMS recomendado pelo National Research Council (2007) para animais com $23 \mathrm{~kg}$ e ganhos de $200 \mathrm{~g}$ por dia. A inclusão do coproduto não limitou o consumo, o que permitiu a expressão do potencial de ganho de peso dos animais.

Diversos fatores podem atuar na inibição do consumo de MS e, entre os fatores inerentes ao alimento, destaca-se o teor de FDN, em razão de sua lenta degradação e baixa taxa de passagem pelo rúmen (Allen, 2000). No presente trabalho, apesar da variação no teor de FDN nas dietas (Tabela 1), em consequência da inclusão do coproduto, não houve alteração no CMS. Um dos fatores que pode explicar tal efeito sobre o consumo é o processamento físico da torta de macaúba. A moagem deste coproduto resulta em partículas com tamanho semelhante ao dos alimentos concentrados padrão, constituídos de milho moído e farelo de soja, o que reduziu a efetividade física da fração fibrosa.

Além disso, mesmo as dietas tendo apresentado valores diferentes nos teores de FDN e EE, estas continham teores de energia semelhante, o que também pode ter contribuído para a semelhança no consumo de MS entre os tratamentos.

O consumo de $\mathrm{PB}$ não foi influenciado pelos níveis de inclusão de TM ( $>>0,05)$. No entanto, o aumento linear $(p<0,01)$ verificado no consumo de $P B$, com 
base no PC e do PM, mostra que houve seleção dos nutrientes proteicos pelos animais, uma vez que as dietas com coproduto continham teores de proteína próximos - de 157,4 a 158,8 $\mathrm{g} \mathrm{kg}^{-1}$ de MS (Tabela 1).

A média de consumo de PB dos tratamentos foi de $174 \mathrm{~g}$ por animal por dia, valor similar ao exigido para ovinos em crescimento (180 g por animal por dia) recomendado pelo National Research Council (2007), enquanto o consumo de PB ( $\left.\mathrm{g} \mathrm{kg}^{-0,75}\right)$, em todos os tratamentos, foi superior ao recomendado $\left(9,6 \mathrm{~g} \mathrm{~kg}^{-0,75}\right)$.

Os consumos de EE e FDN aumentaram $(\mathrm{p}<0,05)$ linearmente com a inclusão do coproduto, o que decorre, em parte, do aumento da concentração desses nutrientes em razão da inclusão da TM nas dietas.

No presente trabalho, o consumo de FDN dos tratamentos com inclusão de TM de 200 e $300 \mathrm{~g} \mathrm{~kg}^{-1}$ de matéria seca foi superior ao citado por Van Soest (1994), de 8 e $12 \mathrm{~g} \mathrm{~kg}^{-1}$ PC para ovinos (Tabela 2).
É importante salientar que teores elevados de FDN na dieta poderiam limitar o CMS (Hübner et al., 2008), o que, provavelmente não ocorreu no presente experimento, pois, o processamento físico da torta de macaúba pode ter reduzido a efetividade da fibra e seu efeito sobre a ingestão de matéria seca.

O consumo médio de CNF foi influenciado $(\mathrm{p}<0,01)$ pela dieta, o que indica efeito linear decrescente dos níveis de TM sobre o consumo de CNF. Esses resultados podem ser justificados pela redução da concentração de CNF nas dietas com a inclusão do coproduto.

Não houve efeito sobre o ganho de peso com a adição de TM na dieta, que variou de 205 a 188 g por animal por dia, o que mostra o potencial de utilização desse coproduto para ovinos em crescimento (Tabela 3).

Segundo Mertens (1994), 60 a 90\% das diferenças do desempenho animal ocorrem em consequência do consumo, e 10 a $40 \%$ em razão da digestibilidade. Normalmente, nos confinamentos, o maior ganho

Tabela 2. Médias e coeficientes de variação quanto ao consumo de matéria seca (CMS), matéria orgânica (CMO), proteína bruta (CPB), extrato etéreo (CEE), fibra em detergente neutro (CFDN) e carboidratos não fibrosos (CCNF), em consequência da inclusão de torta de macaúba nas dietas de cordeiros Santa Inês.

\begin{tabular}{|c|c|c|c|c|c|c|c|}
\hline \multirow[t]{2}{*}{ Variáveis } & \multicolumn{4}{|c|}{ Inclusão de torta de macaúba ( $\mathrm{g} \mathrm{kg}^{-1}$ de matéria seca) } & \multirow{2}{*}{$\begin{array}{l}\mathrm{CV} \\
(\%)\end{array}$} & \multicolumn{2}{|c|}{$P$} \\
\hline & 0 & 100 & 200 & 300 & & Linear & Quadrático \\
\hline CMS (g por dia) & 1093,56 & 1093,30 & 1132,85 & 1179,46 & 11,38 & 0,319 & 0,722 \\
\hline CMS ( $\mathrm{g} \mathrm{kg}^{-1}$ de peso corporal) & 36,52 & 36,40 & 38,00 & 39,88 & 7,27 & 0,053 & 0,441 \\
\hline CMS $\left(\mathrm{g} \mathrm{kg}^{-0,75}\right)$ & 85,29 & 85,16 & 88,67 & 92,95 & 8,14 & 0,073 & 0,489 \\
\hline CMO (g por dia) & 1055,95 & 1047,65 & 1078,94 & 1113,65 & 11,34 & 0,469 & 0,732 \\
\hline $\mathrm{CMO}$ ( $\mathrm{g} \mathrm{kg}^{-1}$ de peso corporal) & 35,27 & 34,88 & 36,19 & 37,66 & 7,18 & 0,130 & 0,450 \\
\hline $\mathrm{CMO}\left(\mathrm{g} \mathrm{kg}^{-0,75}\right)$ & 82,37 & 81,61 & 84,46 & 87,76 & 8,06 & 0,166 & 0,499 \\
\hline CPB (g por dia) & 157,08 & 176,29 & 179,20 & 185,29 & 12,87 & 0,099 & 0,568 \\
\hline CPB $\left(\mathrm{g} \mathrm{kg}^{-0,75)(1)}\right.$ & 12,21 & 13,72 & 14,01 & 14,59 & 9,92 & 0,010 & 0,426 \\
\hline $\mathrm{CEE}(\mathrm{g} \text { por dia })^{(2)}$ & 42,26 & 55,61 & 72,39 & 80,02 & 12,34 & $<0,001$ & 0,458 \\
\hline CFDN (g por dia) $)^{(3)}$ & 313,68 & 366,10 & 446,74 & 489,29 & 11,32 & $<0,001$ & 0,831 \\
\hline CFDN ( g kg$^{-1}$ de peso corporal) ${ }^{(4)}$ & 10,48 & 12,19 & 14,97 & 16,55 & 7,89 & $<0,001$ & 0,901 \\
\hline CCNF $(\text { g por dia })^{(5)}$ & 542,93 & 449,64 & 380,60 & 359,03 & 12,38 & $<0,001$ & 0,190 \\
\hline
\end{tabular}

(1) $\mathrm{y}=0,00743 \mathrm{x}+12,518 ; \mathrm{R}^{2}=89,31 \% .{ }^{(2)} \mathrm{y}=0,13 \mathrm{x}+43,06 ; \mathrm{R}^{2}=98,1 \% .{ }^{(3)} \mathrm{y}=0,6074 \mathrm{x}+312,8 ; \mathrm{R}^{2}=98,6 \% .{ }^{(4)} \mathrm{y}=0,0211 \mathrm{x}+10,40 ; \mathrm{R}^{2}=98,8 \%$. ${ }^{(5)} y=-0,6207 x+526,1 ; R^{2}=93,6 \%$.

Tabela 3. Médias e coeficiente de variação dos pesos vivo inicial (PVI) e final (PVF), ganho médio diário (GMD), ganho de peso vivo total (GPVT) e da conversão alimentar da matéria seca (CAMS), de cordeiros confinados e alimentados com dieta com inclusão de torta de macaúba.

\begin{tabular}{|c|c|c|c|c|c|c|c|}
\hline \multirow[t]{2}{*}{ Variáveis } & \multicolumn{4}{|c|}{ Inclusão de torta de macaúba ( $\mathrm{g} \mathrm{kg}^{-1}$ de matéria seca) } & \multirow{2}{*}{$\begin{array}{l}\text { CV } \\
(\%)\end{array}$} & \multicolumn{2}{|c|}{$P$} \\
\hline & 0 & 100 & 200 & 300 & & Linear & Quadrático \\
\hline PVI (kg) & 23,55 & 24,18 & 23,65 & 24,11 & 4,16 & 0,829 & 0,944 \\
\hline PVF (kg) & 35,86 & 36,16 & 35,85 & 35,38 & 7,65 & 0,836 & 0,840 \\
\hline GMD (g por animal por dia) & 205,28 & 199,72 & 203,33 & 187,78 & 20,24 & 0,570 & 0,794 \\
\hline GPVT (g por dia) & 12,31 & 11,98 & 12,20 & 11,26 & 20,24 & 0,570 & 0,794 \\
\hline CAMS (g de MS por g de peso ganho) $)^{(1)}$ & 5,53 & 5,63 & 5,84 & 6,40 & 8,82 & 0,015 & 0,344 \\
\hline
\end{tabular}

${ }^{(1)} y=0,0028 x+5,43 ; R^{2}=87,7 \%$. MS, matéria seca. 
de peso pode ser obtido como resultado de maior consumo de nutrientes e matéria seca (Barroso et al., 2006). Embora a inclusão do coproduto tenha alterado o consumo de alguns nutrientes (Tabela 2), o aporte energético das dietas foi similar (Tabela 1), o que justifica a semelhança nos ganhos de peso observados.

Não houve efeito $(p>0,05)$ da inclusão da TM sobre o PVF e GPVT. A diferença percentual no GPVT, entre os animais que não receberam o coproduto $\mathrm{e}$ aqueles que receberam a dieta com $300 \mathrm{~g} \mathrm{~kg}^{-1}$ de torta de macaúba, foi de $9,02 \%$, o que mostra o potencial de utilização deste coproduto para cordeiros em confinamento, uma vez que o milho foi o principal ingrediente substituído.

A conversão alimentar variou de 5,5 a 6,4 e foi negativamente $(p<0,05)$ influenciada pelos níveis de TM nas dietas. A diferença na conversão alimentar pode ser justificada pelo aumento no CMS, em razão da inclusão da TM nas dietas ( $\mathrm{p}=0,053)$.

A conversão alimentar é um importante parâmetro a ser utilizado para a avaliação econômica das dietas. No presente trabalho, o efeito linear da inclusão da TM nas dietas sobre a CA mostra que os animais necessitaram consumir maiores quantidades da dieta, para convertê-la em $1 \mathrm{~kg}$ de $\mathrm{PV}$, o que na prática pode aumentar o custo com alimentação dos animais, a depender da relação de preços existentes entre o produto substituído e o coproduto.

Houve redução no custo $\left(\mathrm{R} \$ \mathrm{~kg}^{-1}\right)$ do concentrado com a inclusão da macaúba (Tabela 4). Os valores encontrados para o custo do concentrado $\left(\mathrm{R} \$ \mathrm{~kg}^{-1}\right)$ são semelhantes aos de Barros et al. (2009), que estudaram a rentabilidade da produção de ovinos de corte, em pastagem e em confinamento, no Estado do Paraná. Esses autores verificaram o valor de $\mathrm{R} \$ 0,40 \mathrm{~kg}^{-1}$ para o concentrado fornecido aos animais em terminação.

Tabela 4. Médias de custos do concentrado e da dieta completa (concentrado+volumoso) por $\mathrm{kg}$ de matéria seca (MS), das dietas com inclusão de torta de macaúba (TM).

\begin{tabular}{|c|c|c|c|c|}
\hline \multirow[t]{2}{*}{ Variáveis } & \multicolumn{4}{|c|}{ Inclusão de TM $\left(\mathrm{g} \mathrm{kg}^{-1}\right.$ de MS) } \\
\hline & 0 & 100 & 200 & 300 \\
\hline Concentrado $\left(\mathrm{R} \$ \mathrm{~kg}^{-1}\right)-\mathrm{MS}$ & 0,42 & 0,41 & 0,40 & 0,39 \\
\hline Volumoso $\left(\mathrm{R} \$ \mathrm{~kg}^{-1}\right)-\mathrm{MS}$ & 0,12 & 0,12 & 0,12 & 0,12 \\
\hline Dieta completa $\left(\mathrm{R} \$ \mathrm{~kg}^{-1}\right)-\mathrm{MS}$ & 0,54 & 0,53 & 0,52 & 0,51 \\
\hline Dieta completa $(\mathrm{R} \$ \text { por animal por dia })^{(1)}$ & 0,58 & 0,57 & 0,59 & 0,60 \\
\hline
\end{tabular}

${ }^{(1)}$ Custo em R $\$$ por kg x consumo de matéria seca por dia.
Apesar da redução verificada no custo do concentrado, a inclusão do coproduto aumentou os custos com a dieta total por animal por dia. Esse aumento foi reflexo da conversão alimentar dos animais e das cotações da macaúba e do milho, pois, durante a época de realização do experimento, a macaúba apresentava preço pouco competitivo em comparação ao preço do milho (Tabela 4).

A avaliação da receita bruta, obtida com a venda dos animais vivos e abatidos, indica uma diferença entre os tratamentos de 2,2 e $6,8 \%$, respectivamente, em que o tratamento com inclusão de $100 \mathrm{~g} \mathrm{~kg}^{-1}$ apresentou maior receita bruta, em razão do maior peso vivo final e maior peso da carcaça fria dos animais submetidos a este tratamento (Tabela 5).

A análise da receita líquida, proveniente da venda dos animais vivos e abatidos, mostra que a diferença entre os tratamentos foi de 6,6 e 12,9\%, respectivamente, e que, novamente, o tratamento com inclusão de $100 \mathrm{~g} \mathrm{~kg}^{-1}$ apresentou a maior receita líquida, reflexo da menor despesa com alimentação dos animais submetidos a esse tratamento (Tabela 5). $\mathrm{O}$ tratamento com inclusão de $300 \mathrm{~g} \mathrm{~kg}^{-1}$ de macaúba apresentou receita bruta inferior, além de maior conversão alimentar, o que influenciou a despesa com alimentação e consequentemente a receita líquida. Esse resultado mostra que a viabilidade econômica do uso da TM depende das cotações do coproduto e do milho, uma vez que, embora a inclusão do coproduto tenha reduzido o preço do quilo de concentrado, essa redução foi insuficiente para compensar o aumento na conversão alimentar.

Tabela 5. Médias de receitas bruta (RB) e líquida (RL), obtidas com a venda de cordeiros vivos ou abatidos, das dietas com inclusão de torta de macaúba (TM) por kg de matéria seca (MS).

\begin{tabular}{lcccc}
\hline Variáveis & \multicolumn{4}{c}{ Inclusão de TM $\left(\mathrm{g} \mathrm{kg}^{-1}\right.$ de MS) } \\
\cline { 2 - 5 } & 0 & 100 & 200 & 300 \\
\hline Peso vivo final $(\mathrm{kg})$ & 35,86 & 36,16 & 35,85 & 35,38 \\
Valor pago pelo animal vivo $\left(\mathrm{R} \$ \mathrm{~kg}^{-1}\right)$ & \multicolumn{4}{c}{3,00} \\
RB do animal vivo (R\$ por animal) & 107,70 & 108,60 & 107,70 & 106,20 \\
Peso da carcaça fria (kg) & 16,59 & 17,16 & 16,42 & 16,03 \\
Valor pago pelo animal abatido (R $\left.\$ \mathrm{~kg}^{-1}\right)$ & \multicolumn{5}{c}{7,00} \\
RB do animal abatido (R\$ por animal) & 116,13 & 120,12 & 114,94 & 112,21 \\
Despesa total da alim. por animal $(\mathrm{R} \$)$ & 36,35 & 35,78 & 37,29 & 38,11 \\
RL do animal vivo (R\$ por animal) & 71,35 & 72,82 & 70,41 & 68,09 \\
RL do animal abatido (R\$ por animal) & 79,78 & 84,34 & 77,65 & 74,10 \\
\hline
\end{tabular}

Alim., alimentação.

Pesq. agropec. bras., Brasília, v.47, n.11, p.1663-1668, nov. 2012 


\section{Conclusões}

1. A inclusão de até $300 \mathrm{~g}$ de torta de macaúba por $\mathrm{kg}$ de matéria seca (MS) na dieta não altera o consumo de MS e o desempenho produtivo de cordeiros Santa Inês.

2. Apesar das alterações no consumo de nutrientes e da conversão alimentar, o uso de $100 \mathrm{~g} \mathrm{~kg}^{-1}$ de torta de macaúba apresenta viabilidade econômica para dietas de cordeiros Santa Inês em terminação.

\section{Agradecimentos}

À Cooperativa de Pequenos Produtores Rurais de Riacho D'Antas e Adjacências, pela doação da torta de macaúba; ao Conselho Nacional de Desenvolvimento Científico e Tecnológico, à Fundação de Amparo a Pesquisa do Estado de Minas Gerais, e ao Fundo de Desenvolvimento Científico e Tecnológico do Banco do Nordeste, por apoio financeiro e concessão de bolsas.

\section{Referências}

ALLEN, M.S. Effects of diet on short-term regulation of feed intake by lactating dairy cattle. Journal of Dairy Science, v.83, p.1598-1624, 2000.

BARRETO, S.M.P. Avaliação do valor nutritivo da torta de macaúba (Acrocomia Aculeata (Jacq.) Lodd. ex Mart.) na alimentação de caprinos. 2008. 74p. Dissertação (Mestrado) Universidade Federal de Minas Gerais, Montes Claros.

BARROS, C.S. de; MONTEIRO, A.L.G.; POLI, C.H.E.C.; DITTRICH, J.R.; CANZIANI, J.R.F.; FERNANDES, M.A.M. Rentabilidade da produção de ovinos de corte em pastagem e em confinamento. Revista Brasileira de Zootecnia, v.38, p.2270-2279, 2009 .

BARROSO, D.D.; ARAÚJO, G.G.L. de; SILVA, D.S. da; GONZAGA NETO, S.; MEDINA, F.T. Desempenho de ovinos terminados em confinamento com resíduo desidratado de vitivinícolas associado a diferentes fontes energéticas. Ciência Rural, v.36, p.1553-1557, 2006.
CARDOSO, A.R.; PIRES, C.C.; CARVALHO, S.; GALVANI, D.B.; JOCHIMS, F.; HASTENPFLUG, M.; WOMMER, T.P. Consumo de nutrientes e desempenho de cordeiros alimentados com dietas que contêm diferentes níveis de fibra em detergente neutro. Ciência Rural, v.36, p.215-221, 2006.

FOOD AND AGRICULTURE ORGANIZATION OF THE UNITED NATIONS. FAOSTAT. Available at: <http://faostat.fao. org/site/573/DesktopDefault.aspx?PageID=573\#ancor $>$. Accessed on: 26 Aug. 2012.

HÜBNER, C.R.; PIRES, C.C.; GALVANI, D.B.; CARVALHO, S.; JOCHIMS, F.; WOMMER, T.P.; GASPERIN, B.G. Comportamento ingestivo de ovelhas em lactação alimentadas com dietas contendo diferentes níveis de fibra em detergente neutro. Ciência Rural, v.38, p.1078-1084, 2008.

INSTITUTO BRASILEIRO DE GEOGRAFIA E ESTATÍSTICA. Produção da pecuária municipal - 2010. Disponível em: $<\mathrm{http}: / /$ www.ibge.gov.br/home/estatistica/economia/ppm/2010/default. shtm>. Acessado em: 26 ago. 2012.

MACEDO, C.A.B. de; MIZUBUTI, I.Y.; MOREIRA, F.B.; PEREIRA, E.S.; RIBEIRO, E.L. de A.; ROCHA, M.A. da; RAMOS, B.M. de O.; MORI, R.M.; PINTO, A.P.; ALVES, T.C.; CASIMIRO, T.R. Comportamento ingestivo de ovinos recebendo dietas com diferentes níveis de bagaço de laranja em substituição à silagem de sorgo na ração. Revista Brasileira de Zootecnia, v.36, p.1910-1916, 2007.

MERTENS, D.R. (Ed.). Regulation of forage intake. In: FAHEY JUNIOR, G.C. Forage quality, evaluation and utilization. Winsconsin: American Society of Agronomy, 1994. p.450-493.

NATIONAL RESEARCH COUNCIL. Nutrient requirements of small ruminants. Washington: National Academy of Sciences, 2007. 362p

NUNES, H.; ZANINE, A. de M.; MACHADO, T.M.M.; CARVALHO, F.C. de. Alimentos alternativos na dieta dos ovinos: uma revisão. Archivos Latinoamericanos de Producción Animal, v.15, p.141-158, 2007.

SAEG: sistema para análises estatísticas. Versão 9.1. Viçosa: UFV, 2007. 142p.

SILVA, D.J.; QUEIROZ, A.C. de. Análise de alimentos: métodos químicos e biológicos. 3.ed. Viçosa: UFV, 2002. 235p.

VAN SOEST, P.J. Nutritional ecology of the ruminant. $2^{\text {nd }}$ ed. Ithaca: Cornell University, 1994. 476p.

WEISS, W.P. Estimating the available energy content of feeds for dairy cattle. Journal of Dairy Science, v.81, p.830-839, 1998.

Recebido em 21 de maio de 2012 e aprovado em 4 de setembro de 2012 